\title{
„The King's Speech“ oder „My Castle, my Rules“ - Zur Prozessgestaltung in der Beratung
}

\author{
Thomas Bachmann
}

Eingegangen: 7. Oktober 2020 / Angenommen: 14. November 2020 / Online publiziert: 28. Januar 2021 (C) Der/die Autor(en) 2021

Zusammenfassung Anhand der Art und Weise, wie der Therapeut im Film „,The King's Speech“ (GBR, USA, AUS, 2010) die Arbeitsbeziehung gestaltet, wird die Verantwortungsdynamik in einem ko-kreativen Veränderungsprozess beschrieben und analysiert. Es werden Elemente der Beziehungsgestaltung für Therapie, Coaching und Beratung dargestellt und Handlungsmöglichkeiten für die Auftragsklärung abgeleitet.

Schlüsselwörter Verantwortung · Arbeitsbeziehung · Beziehungsaufbau ·

Auftragsklärung

\section{“The King's Speech" or "My Castle, my Rules"—On process design in consulting}

Abstract Based on the way the therapist in the film "The King's Speech" (GBR, USA, AUS, 2010) shapes the working alliance, the dynamics of responsibility in a co-creative change process is described and analyzed. Elements of relationship design for therapy, coaching and counseling are presented and possible courses of action for clarifying the assignment are derived.

Keywords Responsibility · Working alliance · Establishing relationship · Clarifying assignment

PD Dr. T. Bachmann ( $₫)$

artop GmbH, Christburger Str. 4, 10405 Berlin, Deutschland

E-Mail: bachmann@artop.de

Humboldt-Universität zu Berlin, Berlin, Deutschland 


\section{Handlung}

Wembley 1925. Auf einer Veranstaltung der British Empire Exhibition soll Albert (Colin Firth), Herzog von York, zweiter Sohn von König Georg V., erstmals eine Hörfunkansprache halten. Seine Nervosität und sein Stottern jedoch lassen die Ansprache im Fiasko enden. Nach zahlreichen erfolglosen Therapieversuchen gegen das Stottern, zu denen verschiedene Ärzte, aber auch zwielichtige Heiler und Quacksalber an den Königshof gebracht wurden, wird schließlich der ganz anders arbeitende australische Sprachtherapeut Lionel Logue (Geoffrey Rush) bemüht. Dessen unkonventionelle Herangehensweise irritiert zunächst: Logue weigert sich, in den Palast zu kommen, vielmehr besteht er darauf, die Sitzungen in seiner eigenen Praxis abzuhalten. Er verzichtet dabei auf jegliche höfische Etikette.

Um Albert die Heilbarkeit des Stotterns zu beweisen, lässt er ihn Hamlets Monolog „Sein oder Nichtsein“ laut vorlesen, während dieser über einen Kopfhörer laute Musik hört: Dabei zeichnet er Alberts Stimme direkt auf eine Schallplatte auf. Resigniert bricht Albert mitten im Monolog ab und verlässt die Sitzung, nimmt aber die Aufnahme mit. Wieder zu Hause legt er die von Logue aufgenommene Schallplatte auf und hört sich selbst flüssig und fehlerfrei sprechen. Die Therapie wird schließlich wieder aufgegriffen und führt im weiteren Verlauf der Geschichte zum Erfolg, sodass Albert, dann als George VI. sowohl seine Krönungszeremonie als auch die wichtige Rundfunkansprache an sein Volk zur Kriegserklärung des Vereinigten Königreichs gegen Deutschland 1939 hervorragend meisterte (Wikipedia 2020).

\section{Situation}

Der Film enthält eine Fülle von therapeutischen und beraterischen Interventionen. In der Arbeit von Lionel Logue sind Elemente aus der Hypnotherapie, dem Psychodrama, der Gestalttherapie, der Logotherapie sowie tiefenpsychologische Interventionen zu erkennen. Auch die direkte Unterstützung des Klienten in Bewährungssituationen im Sinne eines Coaching ,on-the-job“ spart der Film nicht aus. Ganz besonders sticht jedoch die Beziehungsgestaltung des Therapeuten mit seinem Klienten hervor.

Der Beginn der Arbeitsbeziehung, wie im Film geschildert, lässt sich gut mit Edgar Scheins Typologie der Beratungsformate beschreiben (Schein 2000, S. 23 ff.): Am königlichen Hof liegt ein Problem vor, und man versucht, durch das Engagement eines Experten, der bestimmte Methoden anwendet, die Symptome zum Verschwinden zu bringen. In diesem Modus behält das Königshaus die Prozesshoheit in der Hand, indem bestimmt wird, wer wie wo und wann seine Verfahren und Methoden an dem jungen stotternden Thronfolger anwenden darf. Tritt keine Besserung ein, kann es der nächste Hoffnungsträger probieren. Doch das Vorgehen bringt keinen Erfolg. Im Gegenteil, eine Verschlimmerung der Symptome tritt ein, und Resignation und Passivität machen sich breit. Es ist wie in dem Märchen von der „Goldenen Gans“: Je mehr die verschiedenen Freier versuchen, die Prinzessin zum Lachen bringen, desto trauriger wird sie. 
Lionel Logue arbeitet völlig anders: Von Anfang an übernimmt er die Prozesshoheit und schafft dadurch einen Raum für Impulse, Reflexionen und Experimente. Dabei setzt er vom ersten Moment an auf die Selbstverantwortung seines Klienten. Dieser muss sich zu seinem Therapeuten begeben, auch wenn er ein Kronprinz ist, er wird nicht wie eine königliche Person behandelt, sondern wie ein ganz normaler Mensch. Lionel Logue dient sich nicht an, er verkauft nichts, er verspricht nichts und er macht sich nicht anheischig. Prinz Albert hat bei ihm keine Privilegien. Viel schlimmer noch: „My castle, my rules“ ist die Ansage von Lionel Logue, mit der er klar macht, wer der Gastgeber ist und nach wessen Regeln gearbeitet wird. Damit überträgt er seinem Klienten die Verantwortung für seine Heilung.

Diese konsequente Form der Beziehungsgestaltung wird dabei durch die spezifische Konstellation der handelnden Personen erleichtert. Der exzentrische Australier Logue trifft auf den royalen Albert: Nationale, kulturelle und soziale Unterschiede begünstigen dabei die Unterschiedsbildung hinsichtlich Rollen, Format und Methodik, die für eine wirksame Arbeitsbeziehung vor allem am Anfang wichtig sind. Diese Unterschiede verschmelzen im Laufe der Handlung, die Beziehung verwandelt sich schließlich in eine Freundschaft.

\section{Intervention}

Fritz B. Simon und Gunthard Weber (2004, S. 24) schreiben über die Verantwortungsdynamik: „Das Maß der Verantwortung in einem Interaktionssystem bleibt konstant. Die Verantwortungsabgabe des einen ist die Verantwortungsübernahme des anderen. Wo nichts ist, kann auch nichts abgegeben werden" (1. Hauptsatz). Und: „Die Verantwortung in einem sozialen System erhält immer der Schnellste“ (2. Hauptsatz).

Die Beziehungsgestaltung in Therapie, Coaching und Beratung ist ein wesentlicher Faktor für den Erfolg. Dabei geht es nicht nur darum, wie es schon hinreichend in der Literatur ausgeführt wurde, eine vertrauensvolle, tragfähige Beziehung auf „Augenhöhe“ zu etablieren. Das ist selbstverständlich. Diese Beziehung muss jedoch über bestimmte Disparitäten verfügen, damit eine Wirksamkeit entsteht. So müssen die Struktur- und die Prozessverantwortung, also der Ablauf, die Rahmenbedingungen, die Arbeitsweise, die eingesetzten Methoden etc. beim Berater, Coach bzw. der Therapeutin liegen, während die Klientinnen Verantwortung für den Inhalt, also ihr Anliegen oder ihr Thema übernehmen müssen (Bachmann und Steinke 2019). Um den Gesetzen der Verantwortungsdynamik gerecht zu werden, muss sichergestellt werden, dass das größere Interesse an der Veränderung beim Klienten liegt. Coaches, Therapeuten und Beraterinnen dürfen nicht „verändern wollen“. Es geht für die Klientinnen darum, sich selbst zu verändern, und dazu müssen sie Verantwortung übernehmen. Denn Verantwortung kann man nur für Prozesse gewährleisten, die man steuern kann (Simon und Weber 2004). Die Erkenntnisse der Systemtheorie zeigen uns, dass autonome Systeme, also Menschen, nicht steuerbar - bestenfalls beeinflussbar - sind. Daher ist es eine unmögliche Aufgabe für Therapeuten, Beraterinnen und Coaches, Verantwortung für die Veränderung ihrer Klienten zu übernehmen. 
Im Fall von Albert bedeutet dies, sich nicht nur aktiv für den Therapieprozess zu entscheiden und seinen Helfer aufzusuchen, sondern vor allem, sich einzubringen und an sich selbst zu arbeiten, indem er beispielsweise traumatische Erlebnisse aus seiner Kindheit durcharbeitet oder die emotional geladene Beziehung zu seinem älteren Bruder thematisiert und im geschützten therapeutischen Raum ausagiert. Damit wird die gemeinsame Arbeit zu einem ko-kreativen Prozess und entzieht sich dem tradierten, instruktiven Beratungsmuster.

\section{Transfer}

Die Beziehungsgestaltung in Coaching und Beratung beginnt bereits mit der Präsentation der eigenen Person und Leistungen. Die eigene Website, Flyer, Profile in Suchportalen und sozialen Medien vermitteln potenziellen Klienten erste Beziehungsbotschaften. Wesentliche Merkmale sind hier, inwieweit sich Coaches und Beraterinnen als Person, mit ihrer Biographie, ihren Prägungen, Erfahrungen und Qualifikationen präsentieren oder sich eher als Vertreter einer bestimmten Methode oder eines Verfahrens (z. B. Reiss-Profile, Aufstellungsarbeit etc.) repräsentieren. Die Kompetenzzuschreibung erfolgt bei Letzteren durch die angebotene Technologie und weniger durch die Person resp. das Selbst des Coachs oder der Beraterin. Damit wird ein Teil der Verantwortung von potenziellen Klientinnen auf die Methode projiziert, die elaboriert und effektiv meist mit Stimmen von Testimonials präsentiert wird. Eine zweite Unterscheidung differenziert, inwieweit durch Coaches oder Berater Erfolgsversprechen kommuniziert werden. Im potenziellen Interaktionssystem mit den Klientinnen wird damit ebenfalls Verantwortung vom Klienten abgegeben und von der Beraterin oder dem Coach übernommen. Nach dem Motto: „Na nun zeigen Sie mal, was Sie drauf haben, Coach!“. Mit den hier kurz beschriebenen Beziehungsbotschaften ist schon einmal ein bedeutender Teil der Verantwortung klientenseitig verloren gegangen, noch bevor das Coaching überhaupt begonnen hat.

Neben den Beziehungsbotschaften, die vor der eigentlichen Interaktion das Miteinander beeinflussen, spielen natürlich die Auftragsklärung im Erstgespräch und die darin vereinbarten Regeln und Verantwortlichkeiten für die Zusammenarbeit eine wesentliche Rolle für die Verantwortungsdynamik. Beispielsweise ist es von Bedeutung, Klienten von Anfang an zu ,Selbstmeldern“ zu machen, - also Coaching nicht aktiv anzubieten, keine Probecoachings zu machen, Klienten keine Coachingstunden ,verkaufen“ zu wollen, Klienten nicht hinterherzutelefonieren oder E-Mails zu schreiben, ob sie wieder einmal eine Stunde nehmen möchten. All diese Hinweise können dabei helfen, dass sich keine „Besucher“ (de Shazer 1989) bei uns einfinden, die passiv abwarten, was wohl passiert.

Der Prozess des Coachings bzw. der Beratung sollte so gestaltet werden, dass Klientinnen von Anfang an Verantwortung für ihren Veränderungsprozess übernehmen müssen und können. Dazu gehört, vorbereitet zu Sitzungen zu erscheinen, Themen und Anliegen zu formulieren, Reflexionsbereitschaft und Offenheit zu zeigen, Hausaufgaben zu erledigen, erarbeitete Lösungen und Vorhaben umzusetzen, sich um Folgetermine zu kümmern u.v.a.m. Im weiteren Verlauf muss die Verantwortungsdynamik vom Coach oder Berater im Auge behalten bzw. an kritischen Punkten 
z.B. durch ,re-contracting“ neu verhandelt werden. All das setzt ein professionelles Selbstverständnis voraus, zu dem u.a. gehört, als Coach oder Beratungsperson aus inhaltlicher und finanzieller Unabhängigkeit heraus agieren zu können.

Originaltitel: The King's Speech

Erscheinungsjahr: 2010

Land: USA, UK, Australien

Regie: Tom Hooper

Besetzung: Colin Firth, Geoffrey Rush, Helena Bonham Carter u. a.

Verfügbarkeit: Amazon: DVD, Amazon prime, Netflix: Streaming

Funding Open Access funding enabled and organized by Projekt DEAL.

Open Access Dieser Artikel wird unter der Creative Commons Namensnennung 4.0 International Lizenz veröffentlicht, welche die Nutzung, Vervielfältigung, Bearbeitung, Verbreitung und Wiedergabe in jeglichem Medium und Format erlaubt, sofern Sie den/die ursprünglichen Autor(en) und die Quelle ordnungsgemäß nennen, einen Link zur Creative Commons Lizenz beifügen und angeben, ob Änderungen vorgenommen wurden.

Die in diesem Artikel enthaltenen Bilder und sonstiges Drittmaterial unterliegen ebenfalls der genannten Creative Commons Lizenz, sofern sich aus der Abbildungslegende nichts anderes ergibt. Sofern das betreffende Material nicht unter der genannten Creative Commons Lizenz steht und die betreffende Handlung nicht nach gesetzlichen Vorschriften erlaubt ist, ist für die oben aufgeführten Weiterverwendungen des Materials die Einwilligung des jeweiligen Rechteinhabers einzuholen.

Weitere Details zur Lizenz entnehmen Sie bitte der Lizenzinformation auf http://creativecommons.org/ licenses/by/4.0/deed.de.

\section{Literatur}

Bachmann, T., \& Steinke, I. (2019). Coaching-Prozesse. In Deutscher Bundesverband Coaching e. V. (Hrsg.), Coaching als Profession. Kompendium mit den Professionsstandards des DBVC (5. Aufl. S. 75-96). Osnabrück: DBVC.

De Shazer, S. (1989). Wege der erfolgreichen Kurztherapie. Stuttgart: Klett-Cotta.

Schein, E. (2000). Prozessberatung für die Organisation der Zukunft: Der Aufbau einer helfenden Beziehung. Bergisch Gladbach: EHP.

Simon, F. B., \& Weber, G. (2004). Vom Navigieren beim Driften. Post aus der Werkstatt der systemischen Therapie. Heidelberg: Carl-Auer.

Wikipedia (2020). The king's speech. https://de.wikipedia.org/wiki/The_King's_Speech. Zugegriffen: 5. Okt. 2020. 


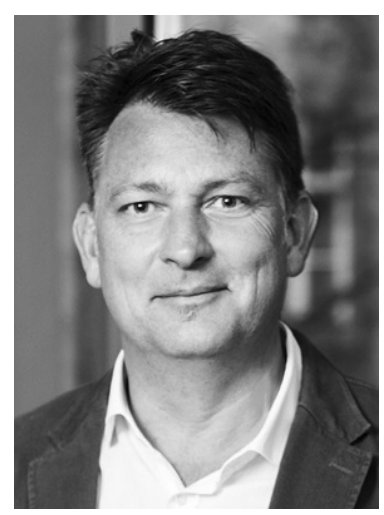

PD Dr. Thomas Bachmann Dipl.-Psych., Gründungsmitglied und Partner der artop GmbH; Studium der Arbeits- und Organisationspsychologie, Klinische Psychologie sowie Informatik, wissenschaftl. Mitarbeiter am Lehrstuhl für Methodenlehre und Statistik, 1997 Promotion in der Kognitiven Psychologie, 2019 Habilitation u. a. zu Systemtheorie und Gestalttherapie. Seit 1993 Berater, Trainer und Coach (DBVC, ICF), seit 2001 Ausbilder und Lehrtrainer für Coaches und Berater. 